Commun. math. Phys. 31, 259--264 (1973)

(C) by Springer-Verlag 1973

\title{
There are no Goldstone Bosons in Two Dimensions *
}

\author{
Sidney Coleman $\star \star$ \\ Joseph Henry Laboratories, Princeton University, Princeton. New Jersey, USA
}

Received February 1, 1973

\begin{abstract}
In four dimensions, it is possible for a scalar field to have a vacuum expectation value that would be forbidden if the vacuum were invariant under some continuous transformation group. even though this group is a symmetry group in the sense that the associated local currents are conserved. This is the Goldstone phenomenon, and Goldstone's theorem states that this phenomenon is always accompanied by the appearance of massless scalar bosons. The purpose of this note is to show that in two dimensions the Goldstone phenomenon can not occur; Goldstone's theorem does not end with two alternatives (either manifest symmetry or Goldstone bosons) but with only one (manifest symmetry).
\end{abstract}

\section{Introduction and Conclusions}

One of the oldest versions of Goldstone's theorem [1] is this: Let $\phi$ be a local scalar field, and let $j_{\mu}$ be a local conserved vector [2] current,

$$
\hat{\partial}^{\mu} j_{\mu}=0 .
$$

Define the scalar field $\delta \phi$ by

$$
\delta \phi(y)=i \int d^{3} \boldsymbol{x}\left[j_{0}\left(x_{0}, \boldsymbol{x}\right), \phi(y)\right] .
$$

(Because of locality - the statement that the fields and currents commute at space-like separations - the integrand always vanishes for sufficiently large $x$; thus the integral always converges. Also, as a consequence of Eq. (1), this object is independent of $x_{0}$ and defines a scalar field [3].) Then, assuming the usual axioms of field theory [4], either (1) the vacuum expectation value of $\delta \phi$ vanishes, or (2) the theory contains a massless scalar meson (the Goldstone boson).

(Formally, $\delta \phi$ is the commutator of $\phi$ with the global conserved charge associated with the local conserved current, and the nonvanishing of its vacuum expectation value implies that the charge does not annihilate the vacuum, or (formally equivalently) that the vacuum

\footnotetext{
* Work supported by the National Science Foundation under Grant No. 30819X and Grant No. 30738X.

$\star \star$ Permanent address: Lyman Laboratory. Harvard University, Cambridge, Massachusetts 02138.
} 
is not invariant under the unitary transformation obtained by exponentiating the charge. This situation is what is usually called spontaneous symmetry breakdown.)

The aim of this note is to demonstrate, that, in a space-time of two dimensions, the corresponding assumptions lead to a strikingly different result: The second alternative is impossible; the vacuum expectation value of $\delta \phi$ always vanishes.

Several comments should be made:

(1) The proof of the result depends critically on certain very special properties of two-dimensional kinematics. There is no reason to believe it casts any doubt upon the occurence of spontaneous symmetry breakdown and the appearance of Goldstone bosons in four-dimensional field theories.

(2) The appearance of vacuum expectation values for scalar fields is a special case of spontaneous symmetry breakdown. There exists a (at least apparently) much more general definition of spontaneous symmetry breakdown - the existence of automorphisms of the algebra of local observables that are not unitarily implementable [5]. I believe it should be possible to generalize the result to this case, but I have not done so.

(3) The result is for continuous symmetries only; it says nothing about the spontaneous breakdown of discrete symmetries, which, even in four dimensions, is not associated with the appearance of Goldstone bosons. This has an analogy in statistical mechanics: the two-dimensional Ising model displays spontaneous magnetization, but the two-dimensional isotropic Heisenberg model does not [6].

(4) Likewise, the result says nothing about the Goldstone-Higgs [7] phenomenon, in which gauge fields prevent the appearance of Goldstone bosons. (The Goldstone theorem is avoided because it is impossible to quantize the gauge fields in a gauge in which all of the axioms are obeyed simultaneously.) Goldstone-Higgs spontaneous symmetry breakdown may well occur even in two dimensions [8].

(5) This work was stimulated by the work of Baumel [9]. Using the methods of constructive field theory, Baumel was able to show that, in the Euclidean lattice approximation, symmetry breakdown did not occur for certain two-dimensional field theories for which semi-classical reasoning would indicate its occurence. This work is an attempt to understand and extend the results of Baumel; the reader should not be misled by accidents of priority of publication into thinking otherwise.

\section{The Central Idea of the Proof}

It is easy to see that there is something special about two dimensions by considering the simplest example of the Goldstone phenomenon, a free 
massless scalar field. The field equation is

The conserved current is

$$
\square^{2} \phi=0 \text {. }
$$

and

$$
j_{\mu}=\partial_{\mu} \phi
$$

$$
\delta \phi=1 \text {. }
$$

Unfortunately, this example breaks down in two dimensions, for in two dimensions there is no free massless scalar field theory [10]. The easiest way to see this is to study the two-point function,

$$
\langle 0|\phi(x) \phi(0)| 0\rangle=\int \frac{d^{2} k}{(2 \pi)} e^{i k \cdot x} \cdot \delta\left(k^{2}\right) \theta\left(k_{0}\right) .
$$

Alas, the right hand side of this equation is ill-defined; if we perform the $k_{0}$ integration, we obtain

$$
\int \frac{d k_{1}}{2 \pi\left|k_{1}\right|}\left(\cos k_{1} x_{1}\right) e^{-i\left|k_{1}\right| x_{0}}
$$

which is divergent at the origin.

Thus, for scalar fields, a delta-function singularity on the light-cone in momentum space is forbidden in two dimensions. This will be the central idea of the proof; we will find that, for $\delta \phi$ to have a non-zero vacuum expectation value, such a forbidden singularity must be present in the two-point function for $\phi$.

\section{A Lemma}

A preliminary result is needed to put the remarks of the last paragraph in usable form. Let us define a peaked test function (of a single variable, $x$ ), as a test function, $f(x)$, with compact support such that

$$
f(x) \leqq f(y) \quad \text { if } \quad x / y \geqq 1 .
$$

In other words, a peaked function is a positive function that is monotone decreasing as one moves away from the origin in either direction [11]. Let us also define, in two-dimensional momentum space, the variables

$$
k_{ \pm}=k_{0} \pm k_{1} \text {. }
$$

Under a Lorentz transformation of rapidity $\alpha$,

$$
k_{ \pm} \rightarrow e^{ \pm \alpha} k_{ \pm} .
$$

Lemma. Let $F\left(k_{+}, k_{-}\right)$be a positive Lorentz-invariant distribution, and let $f\left(k_{-}\right)$be a peaked test function. Then

$$
\lim _{\lambda \rightarrow \infty} \int d k_{-} f\left(\lambda k_{-}\right) F\left(k_{+}, k_{-}\right)=c \delta\left(k_{+}\right),
$$


with $c$ some positive number. (Note that if $\delta\left(k^{2}\right)$ were a distribution in two-dimensional space, it would be a counterexample to this lemma.)

Proof. For any $\lambda$, the object inside the limit is a positive distribution; by Eq. (8), it is a monotone decreasing function of $\lambda$; thus the limit exists and is a positive linear functional. By applying a Lorentz transformation, Eq. (10), we see that the limit functional assigns the same value to $g\left(e^{\alpha} k_{+}\right)$ as to $g\left(k_{+}\right)$, where $g$ is any test function. By choosing $g$ to be a peaked function, we easily find that the support of the limit functional is restricted to the origin.

Let us define

\section{The Proof}

and

$$
\begin{aligned}
F(k) & =\int d^{2} x e^{i k \cdot x}\langle 0|\phi(x) \phi(0)| 0\rangle, \\
F_{\mu}(k) & =\int d^{2} x e^{i k \cdot x}\left\langle 0\left|j_{\mu}(x) \phi(0)\right| 0\right\rangle, \\
F_{\mu v}(k) & =\int d^{2} x e^{i k \cdot x}\left\langle 0\left|j_{\mu}(x) j_{v}(0)\right| 0\right\rangle .
\end{aligned}
$$

Current conservation, Eq. (1), implies that

whence,

$$
k^{\mu} F_{\mu}(k)=0,
$$

$$
F_{\mu}(k)=\sigma k_{\mu} \delta\left(k^{2}\right) \theta\left(k_{0}\right)+\varepsilon_{\mu \nu} k^{v} \varrho\left(k^{2}\right) \theta\left(k_{0}\right) .
$$

where $\sigma$ is a number and $\varrho$ a scalar distribution. [The first term is a welldefined distribution despite our previous observations; the extra factor of $k$ kills the singularity in Eq. (7).] A simple computation shows that

$$
\begin{aligned}
\langle 0|\delta \phi(0)| 0\rangle & =i\left\langle 0\left|\int d x_{1}\left[j_{0}\left(x_{0}, x_{1}\right), \phi(0)\right]\right| 0\right\rangle \\
& =i \sigma / 4 \pi .
\end{aligned}
$$

The second (parity-violating) term in Eq. (13) does not contribute to the integral; it is odd in $x_{1}$.

For any test function $h(x)$, and for any two numbers $a$ and $b$, the state

$$
\int d^{2} x h(x)\left[a j_{0}(x)+b \phi(x)\right]|0\rangle
$$

must have positive norm. This implies that $F_{00}$ and $F$ are positive distributions, and also that

$$
\begin{gathered}
{\left[\int d^{2} k F(k)|\tilde{h}(k)|^{2}\right]\left[\int d^{2} k F_{00}(k)|\tilde{h}(k)|^{2}\right]} \\
\geqq\left|\left[\int d^{2} k F_{0}(k)|\tilde{h}(k)|^{2}\right]\right|^{2},
\end{gathered}
$$

where $\tilde{h}$ is the Fourier transform of $h$.

Now let us choose

$$
\tilde{h}(k)=f\left(\lambda k_{-}\right) g\left(k_{+}\right)+f\left(\lambda k_{+}\right) g\left(k_{-}\right),
$$


where $f$ is a peaked test function and $g$ is a test function whose support does not include the origin. Since $h$ is parity even, the parity-violating second term in Eq. (13) does not contribute to

$$
\int d^{2} k F_{0}(k)|\tilde{h}(k)|^{2}=\sigma|f(0)|^{2} \int d k_{+}\left|g\left(k_{+}\right)\right|^{2} .
$$

Note that this is independent of $\lambda$.

On the other hand, for sufficiently large $\lambda$, the two terms in Eq. (16) have no common support; thus, each of the integrals on the left-hand side of the inequality (15) breaks up into the sum of two terms. By the lemma and the assumed support properties of $g$, the first integral vanishes as $\lambda$ goes to infinity. Because $F_{00}$ is a positive distribution, the second integral is monotone decreasing. Thus,

$$
\sigma=0
$$

and the vacuum expectation value of $\delta \phi$ vanishes. This completes the proof.

Acknowledgment. This work would have been neither initiated nor completed were it not for conversations with Arthur Wightman.

\section{References and Notes}

1. Goldstone, J., Salam, A., Weinberg, S.: Phys. Rev. 127, 965 (1962).

2. Parity conservation is not assumed. Thus, "vector" refers only to transformation properties under the connected Lorentz group, as does "scalar".

3. Actually, the right-hand side of Eq. (2) is intolerably crudely defined. For a proper definition, see Kastler, D., Robinson,D., Swieca, A.: Commun. math. Phys. 3, 151 (1966). The use of the proper definition does not affect the proof given in the text, except by making some of the equations look more complicated.

4. As stated, for example. In: Streater, R., Wightman, A.: TCP, Spin and Statistics, and All That. New York: R. A. Benjamin 1964.

5. This is the procedure of Kastler, Robinson, and Swieca (Ref. 3). The desired generalization has been proved by L. Landau (private communication).

6. Mermin, N.D., Wagner, H.: Phys. Rev. Letters 17, 1133 (1966).

7. Englert, F., Brout, R.: Phys. Rev. Letters 13, 321 (1964). - Higgs, P.: Phys. Letters 12, 132 (1964). - Guralnik, G., Hagen, C., Kibble, T.: Phys. Rev. Letters 13, 585 (1964). Higgs, P.: Phys. Rev. 145, 1156 (1966). - Kibble, T.: Phys. Rev. 155, 1554 (1967).

8. At least formally, it is possible to set up the theory of gauge fields in two dimensions. The gauge fields have no dynamical degrees of freedom, but do create a Coulomb interaction between charge densities. (This is most easily seen by quantizing the theory in axial gauge.)

9. Private communication (through A. Wightman).

10. This is an old observation. Schroer, B.: Fortschr. der Physik 11, 1 (1963) and Wightman,A.: in High Energy Electromagnetic Interactions and Field Theory, ed. by Levy, M.: New York: Gordon and Breach 1967.

This statement should not be taken to mean that there are no zero mass scalar particles in two dimensions. Indeed, if one defines "particle" in the usual way, as a normalizable 
eigenstate of $P_{\mu} P^{\mu}$, the usual two-dimensional theory of massless Dirac fields contains massless scalar particles; these are states of one fermion and one antifermion, both in normalizable states moving to the left. It is a peculiarity of massless two-dimensional kinematics that, despite the fact that this is a normalizable two-particle state in Fock space, it is still an eigenstate of $P_{\mu} P^{\mu}$. Consistent with the remarks above, though, the field $: \bar{\psi} \psi:$, whose two-point function one might expect to possess a delta-function singularity because of the existence of these states, has in fact zero amplitude for creating these states from the vacuum.

11. In this paragraph, and in the sequel, "positive" means non-negative, not positivedefinite. Also, "distribution" means tempered distribution, "test function" means an infinitely differentiable function of fast decrease, and a "positive distribution" means one which assigns positive values to positive test functions.

\author{
S. Coleman \\ Joseph Henry Laboratories \\ Princeton University \\ Princeton, N.J. 08540, USA
}

